\title{
The Magic Angle: a solved mystery
}

\author{
B. Jouffrey ${ }^{a}$ P. Schattschneider ${ }^{\mathrm{a}, \mathrm{b}}$ C. Hébert ${ }^{\mathrm{b}}$ \\ ${ }^{a}$ LMSS-Mat, CNRS-URA 850, École Centrale Paris, Gde Voie des Vignes, \\ F-92295 CHÂTENAY-MALABRY, France \\ ${ }^{\mathrm{b}}$ Institut für Festkörperphysik, Technische Universität Wien, A-1040 WIEN
}

\begin{abstract}
We resolve the mysterious discrepancy between the experimental magic angle in EELS - $\sim 2 \vartheta_{E}$ - and the quantum mechanical prediction of $\sim 4 \vartheta_{E}$. A relativistic approach surpassing the usually applied kinematic correction yields a magic angle close to the experimental value. The reason is that the relativistic correction of the inelastic scattering cross section in anisotropic systems is significantly higher than in isotropic ones.
\end{abstract}

Key words: ELNES, Magic angle, anisotropy

\section{Introduction}

Orientation-dependent Energy Loss Near Edge Structure (ELNES) is used for the study of the anisotropy of the electronic structure[1-4]. On the other hand, electronic anisotropy may cause errors in the investigation of phases by fingerprinting. Similar errors occur in the determination of the sp2/sp3 ratio in carbon. the effect of anisotropy is cancelled when using the "magic" collection angle $[5,1,6]$. The theoretical value for the magic angle is $\sim 4 \theta_{E}$. Daniels et al published a value of $2 \theta_{E}$ but their derivation was shown to contain an error[7]. The only detailled experimental work is due to Daniels et al. with a value of $2 \theta_{E}$. Different explanation were proposed for the discrepancies between experiment and thory like non dipole transitions, intensities coming from other Bragg spots or channeling effects [7].

All the published work is based on the semi relativistic approach [8] which changes the angular scattering distribution in isotropic systems by less than $10 \%$ at $200 \mathrm{kV}$. We show in the following that this is not the case for anisotropic systems. A correnct relativistic treatment yields a magic angle which is considerably smaller than the classical one and comes close to the experimental value. 


\section{Non-relativistic differential cross section}

The double differential scattering cross section for inelastic electron scattering in the classical limit reads

$$
\frac{\partial^{2} \sigma}{\partial E \partial \Omega}=\frac{k^{\prime}}{k_{0}} \frac{e^{4}}{Q^{2}} S\left(\mathbf{q}_{0}, E\right)
$$

where $S$ is the dynamic form factor (DFF).

The magic collection angle for medium voltage $(200-300 \mathrm{kV})$ is of the order of mrad. The corresponding q-values are then $k_{0} \vartheta \sim 10^{9} / \mathrm{m}$, and $q a_{0} \ll 1$ where $k_{0}$ is the incident electron's wave number, and $a_{0}$ is the Bohr radius. The dipole approximation is therefore valid. $k^{\prime}$ is the outgoing electron's wave number, considering $k^{\prime} / k_{0} \simeq 1$ we can then write for the DFF

$$
S(\mathbf{q}, E) \doteq \sum_{i, f}|\langle i|\mathbf{q} \mathbf{r}| f\rangle|^{2} \delta\left(E-E_{i}+E_{f}\right)
$$

$\mathrm{E}$ is the energy loss of the probe electron, the sum is taken over initial and final states $\mathrm{i}, \mathrm{f}$ of the scatterer with energies $E_{i}$ and $E_{f} . Q$ is the convenient abbreviation

$$
Q=\frac{4 \pi \epsilon_{0}\left(\hbar q_{0}\right)^{2}}{2 m}
$$

and $e, m$ are the charge and mass of the electron.

From the scattering geometry we can express $\mathbf{q}_{\mathbf{0}}$ in cylindrical coordinates which relate to the azimuth $\varphi$ and to the scattering angle $\vartheta$ as

$$
\mathbf{q}_{\mathbf{0}}=\left(\begin{array}{c}
k_{0} \sin \vartheta \sin \varphi \\
k_{0} \sin \vartheta \cos \varphi \\
k_{0} \vartheta_{E}
\end{array}\right)
$$

The incident electron's trajectory is parallel to the cylinder axis. For small scattering angles we can replace $\sin \vartheta$ by $\vartheta$. The classical characteristic scattering angle is then $\vartheta_{E}=E / 2 E_{0} .\left(\vartheta=\vartheta_{E}\right.$ defines the HWHM of the Lorentzian angular scattering distribution.)

$Q$ can be interpreted as the kinetic energy of an electron with momentum $\hbar q$, i. e. the energy which a photoelectron would have that was free and at rest before the scattering.

The kinematically corrected expression for the characteristic scattering angle accounts for the relativistic increase of mass - given by the relativistic factor $\gamma=1 / \sqrt{1-\beta^{2}}$ with velocity $v=\beta c$ of the probe electron. The kinetic energy 
$E_{0}=p_{0}^{2} /(2 \gamma m)$ deviates then from the classical quadratic $p^{2}$ behaviour. An elegant derivation was given by Fano[9]: For small energy losses, $E \ll E_{0}$ we have for forward scattering

$$
\left.\Delta p \doteq \frac{d p}{d E}\right|_{E_{0}} E
$$

wherefrom

$$
\vartheta_{E}:=\frac{\Delta p}{p_{0}}=\frac{E}{p_{0} v_{0}}=\frac{E}{\gamma m v_{0}^{2}} .
$$

or[10] $\vartheta_{E}=E /(2 \gamma T)$ with the classical kinetic energy $T=m v_{0}^{2} / 2$ of the incident electron. This is a good approximation for incident energies up to 200

- $300 \mathrm{keV}$. With this correction the magic angle is found to be $3.97 \vartheta_{E}[1]$.

\section{Relativistic Correction}

In fact, the kinematic correction does not account for magnetic forces between probe and target electrons, and for retarded potentials which should be used for high electron velocities. One should also use Dirac wave functions but this does not make any difference when the incident electron is not spin polarized.

It was shown[11]that the magnetic forces shorten the apparent length of the vector $\mathbf{q}$ in the incident direction to

$$
\mathbf{q}=\mathbf{q}_{\mathbf{0}}-\mathbf{v} \frac{\Delta E}{\hbar c^{2}}
$$

where $\mathbf{v}$ is the incident electron's velocity, and $\mathbf{q}_{\mathbf{0}}=\mathbf{k}_{f}-\mathbf{k}_{0}$. Use of Eq.1 for the z-component yields

$$
\mathbf{q}^{\prime}=\left(\begin{array}{c}
k_{0} \sin \vartheta \sin \varphi \\
k_{0} \sin \vartheta \cos \varphi \\
k_{0} \vartheta_{E}\left(1-\beta^{2}\right)
\end{array}\right)
$$

Retarded potentials cause a replacement of $Q$ by[11]

$$
Q=\frac{4 \pi \epsilon_{0}}{2 m}\left(\hbar^{2} q^{2}-\frac{\Delta E^{2}}{c}\right) \text {. }
$$

As noted by Gould[12] $Q$ is the square of a relativistic 4-vector and is as such Lorentz invariant.

It is convenient to choose a coordinate system $\left(x_{1}, x_{2}, x_{3}\right)$ in which the target has the highest possible symmetry. For a uniaxial anisotropic medium, the 
anisotropy axis can be $x_{3}$, say. We decompose the product $\mathbf{q r}$ in the dipole matrix element of the DFF into components of this natural coordinate system as

$$
S(\mathbf{q}, E) \doteq \sum_{j, l} \sum_{i, f} q_{j} q_{l}\left\langle i\left|x_{j}\right| f\right\rangle\left\langle f\left|x_{l}\right| i\right\rangle \delta\left(E-E_{i}+E_{f}\right) .
$$

It can be shown that under very general conditions ${ }^{1}$ the cross terms in the sum vanish. The DFF is therefore a quadratic form. Abbreviating

$$
\sum_{i, f}\left\langle i\left|x_{j}\right| f\right\rangle:=\left\langle x_{j}\right\rangle
$$

the DFF is

$$
S(\mathbf{q}, E) \doteq \sum_{j} q_{j}^{2}\left|\left\langle x_{j}\right\rangle\right|^{2} \delta\left(E-E_{i}+E_{f}\right)
$$

\section{Rotation matrix}

The specimen's $x_{3}$-axis be tilted by $\Gamma$ relative to the Cartesian system $\xi, \eta, \zeta$ in which the vector $\mathbf{q}^{\prime}$, eq. 2 is defined. Choosing the $\xi=x_{1}$ axis as rotation axis, we have then

$$
\begin{aligned}
& q_{1}=\xi \\
& q_{2}=\eta \cos \Gamma-\zeta \sin \Gamma \\
& q_{3}=\eta \sin \Gamma+\zeta \cos \Gamma
\end{aligned}
$$

or in matrix algebra

$$
\mathbf{q}=\mathbf{T}(\Gamma) \cdot \mathbf{q}^{\prime}
$$

with rotation matrix $\mathbf{T}$.

In anisotropic systems, at least one of the matrix elements $\left\langle x_{j}\right\rangle$ is different from the others, by definition. For a uniaxial system, $\left\langle x_{3}\right\rangle \neq\left\langle x_{2}\right\rangle=\left\langle x_{1}\right\rangle$. In general, these will be functions of energy loss; they define two linearly independent basis functions. By reference to the case of graphite, we call them

$$
\begin{aligned}
\sigma^{*}(E) & :=\sum_{i, f}\left|\left\langle i\left|x_{1}\right| f\right\rangle_{f}\right|^{2} \delta\left(E-E_{i}+E_{f}\right) \\
\pi^{*}(E) & :=\sum_{i, f}\left|\left\langle i\left|x_{3}\right| f\right\rangle_{f}\right|^{2} \delta\left(E-E_{i}+E_{f}\right) .
\end{aligned}
$$

\footnotetext{
$\overline{1}$ When the scatterer has at least two perpendicular mirror planes and is nonmagnetic[13].
} 
With these prerequisites we can now integrate the differential cross section up to the collection angle $\alpha$, so as to obtain the partial cross section

$$
\begin{aligned}
\Delta \sigma(\Gamma, \alpha, E) & =e^{4} \sigma^{*}(E) \int_{0}^{2 \pi} d \varphi \int_{0}^{\alpha} \frac{q_{1}^{2}(\vartheta, \varphi)+q_{2}^{2}(\Gamma, \vartheta, \varphi)}{Q^{2}} \vartheta d \vartheta \\
& +e^{4} \pi^{*}(E) \int_{0}^{2 \pi} d \varphi \int_{0}^{\alpha} \frac{q_{3}^{2}(\Gamma, \vartheta, \varphi)}{Q^{2}} \vartheta d \vartheta
\end{aligned}
$$

Inserting $\mathbf{q}^{\prime}$ from eqs. $(2,3)$, and using the basis functions Eq. (5) the $\varphi$ integral can be performed first. The terms containing $\sin \varphi, \cos \varphi$ or $\sin \varphi \cos \varphi$ vanish according to orthogonality. The result has a remarkable structure. It is a linear combination of the two basis functions $\sigma^{*}$ and $\pi^{*}$ with $\Gamma$-dependent coefficients:

$$
\begin{aligned}
\Delta \sigma(\Gamma, \alpha, E) & \propto \sigma^{*}(E) \cdot\left[b(\alpha) \cos ^{2}(\Gamma)+c(\alpha) \sin ^{2}(\Gamma)+a(\alpha)\right]+ \\
& +\pi^{*}(E) \cdot\left[c(\alpha) \cos ^{2}(\Gamma)+b(\alpha) \sin ^{2}(\Gamma)\right]
\end{aligned}
$$

with

$$
\begin{aligned}
a(\alpha) & :=\pi \int_{0}^{\alpha} \frac{\vartheta^{3}}{Q^{2}} d \vartheta \\
b(\alpha) & :=a(\alpha) \\
c(\alpha) & :=2 \pi \int_{0}^{\alpha} \frac{\left(1-\beta^{2}\right)^{2} \vartheta_{e}^{2} \vartheta}{Q^{2}} d \vartheta
\end{aligned}
$$

Expression 7 is independent of the tilt angle $\Gamma$ if $\sigma^{*}(E)=\pi^{*}(E)$ or if

$$
b=c .
$$

The first condition means isotropy and is of no importance here. Fig. 1 shows the functions $\mathrm{c}$ (thick line) and $\mathrm{b}=\mathrm{a}$ as a function of $\alpha$, for $200 \mathrm{keV}$ incident energy. Abscissa in units $\vartheta_{E}$. (The dashed lines are for non-relativistic treatment). The crossing of the curves defines the magic angle.

Fig. 2 shows the magic angle as a function of incident energy. At $200 \mathrm{keV}$ it is found numerically to be $1.46 \vartheta_{E}$.

\section{Conclusion}

The quantum mechanical prediction with relativistic correction for the magic angle is $1.46 \vartheta_{E}$ at $200 \mathrm{kV}$, a value very close to the experiments. Daniels et 


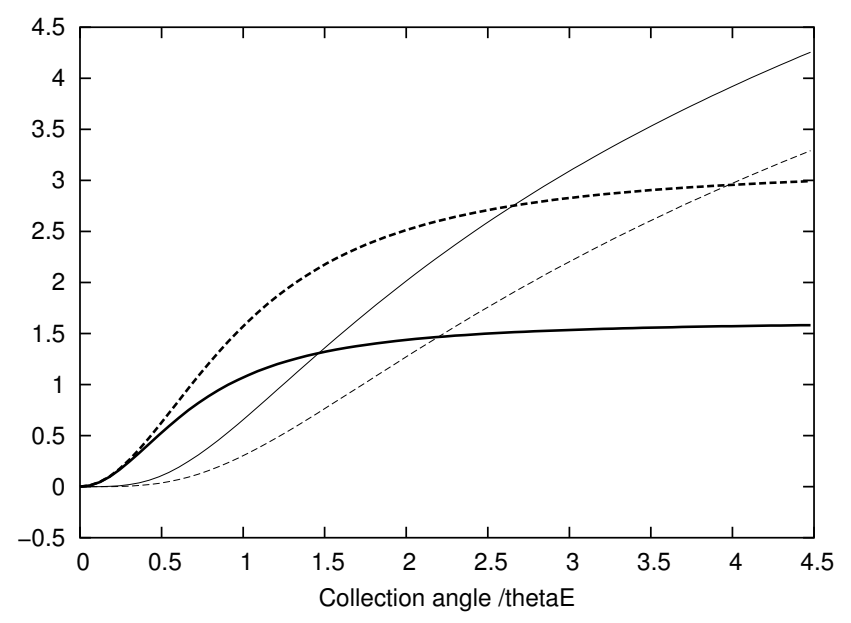

Fig. 1. Prefactors $\mathrm{b}$ and $\mathrm{c}$ of the functions $\sigma^{*}$ and $\pi^{*}$, Eq. 5. thick lines: c. The crossing defines the magic angle. Dashed: non-relativistic factors. Abscissa in units $\vartheta_{E}$.

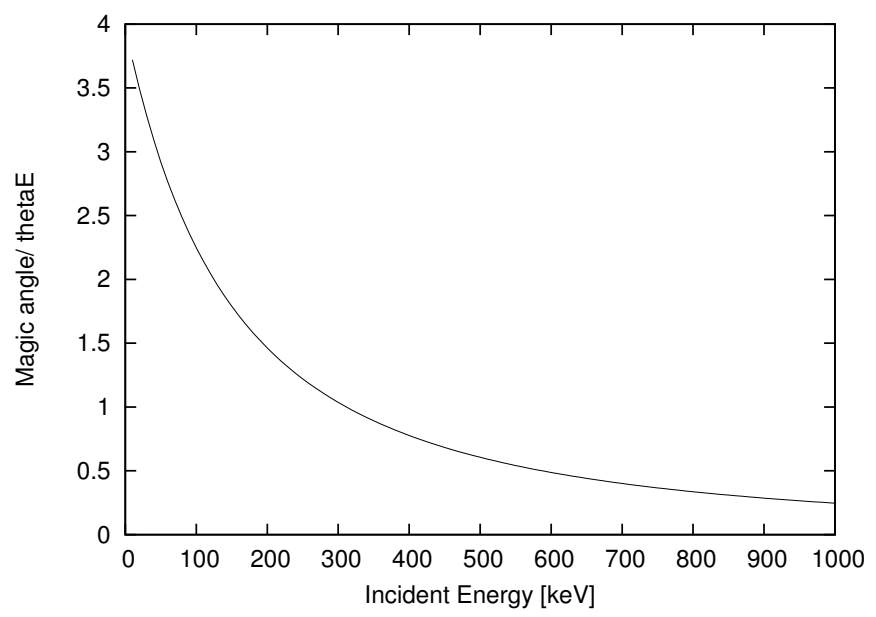

Fig. 2. Magic angle (in units $\vartheta_{E}$ ) as a function of incident energy. Abscissa in keV.

al. found a magic angle close to $2 \vartheta_{E}$ for a variety of anisotropic materials and edges (C-K edge in graphite, B-K and N-K edges in h-BN and B-K edge in $\mathrm{MgB}_{2}$ ). More precisely, their values vary between 1.7 and $2.1 \vartheta_{E}$. A preliminary experimental verification with graphite done on a TECNAI F20 resulted in a magic angle of $1.7 \pm 0.3 \vartheta_{E}$. A detailled experiment with different high voltages is under way.

The relativistic magic angle is 2.7 times smaller than the classical one. This is surprising at first glance since the relativistic correction to the inelastic cross sections at $200 \mathrm{kV}$ is only $\sim 10 \%$. So one would expect a similarly small effect on the magic angle. However, since the corrections act differently in the optical axis and in the plane perpendicular to this axis, the partial cross sections for transitions to $\pi^{*}$ and to $\sigma^{*}$ are also changed differently. In fact, the former ones 
are decreased whereas the latter ones are increased in strength. This becomes visible in the expressions for a, b, c, and in fig. 1 . The relativistic correction in $Q$ has decreased the common denominator, thus increasing a and b. On the other hand, the nominator in the prefactor for the $\zeta$ axis is smaller by a factor $1 /\left(1-\beta^{2}\right)^{2}=\gamma^{4}$ which makes $\sim 3.75$ at $200 \mathrm{kV}$ primary voltage!

Apart from experimental uncertainties, several effects can explain the remaining disagreement between experiment and theory. First, coupling to diffuse quasi-elastic and to Bragg scattering can change the apparent fine structure, and consequently change the measured magic angle. Errors in the calibration of the camera length may contribute with $\pm 3 \%$. Finally, channeling effects in a zone axis orientation which was exactly the experimental condition for the non-tilted series - and non-dipole transitions may play a minor role. We do not consider them as important in explaining the differences between theory and experiment. There is however one theoretical aspect that might explain the disagreement: As pointed out by Fano[9] a proper relativistic approach should include the exchange of virtual photons (described by a transverse electromagnetic field) whereas the present approach is based on purely longitudinal electric field components. The effect is important when the velocity of the electron comes close to the light velocity in the medium since the virtual photons then become real and cause Cerenkov radiation. Since the dielectric function of graphite for K-losses is close to 1 we are probably far off such conditions. It remains to be seen whether inclusion of the virtual photon improves the theoretical prediction.

\section{Acknowledgements}

This work was partially supported by the European Commission, FP6-2003NEST-A, contract nr. 508971 (CHIRALTEM).

\section{References}

[1] C. Souche, B. Jouffrey, G. Hug and M. Nelhiebel, Micron 29 (1998) 419.

[2] C.

Hébert-Souche, P.-H. Louf, P. Blaha, M. Nelhiebel, J. Luitz, P. Schattschneider, K. Schwarz and B. Jouffrey, Ultramicroscopy 83 (2000) 9.

[3] K. Lie, R. Høier and R. Brydson, Physical Review B 61 (2000) 1786.

[4] D. Su, C. Hébert, M. Willinger and R. Schlögl, Micron 34 (2003) 227.

[5] N. K. Menon and J. Yuan, Ultramicroscopy 74 (1998) 83. 
[6] H. Daniels, A. Brown, A. Scott, T. Nichells, B. Rand and R. Brydson, Ultramicroscopy 96 (2003) 523.

[7] C. Hébert, P. Schattschneider and B. Jouffrey, Ultramicroscopy (accepted).

[8] H. A. Bethe, Ann. Phys 5 (1930) 325.

[9] U. Fano, Physical Review 102 (1956) 385.

[10] R. F. Egerton, Electron Energy Loss Spectroscopy in the electron microscope (Plenum Press, 1986).

[11] H. Bethe, Quantentheorie, volume 24 I of Handbuch der Physik (Springer, Berlin, 1933), 2nd edition 495-506.

[12] R. Gould, Physica 62 (1972) 555.

[13] M. Nelhiebel, P.-H. Louf, P. Schattschneider, P. Blaha, K. Schwarz and B. Jouffrey, Physical Review B 59 (1999) 12807. 